\title{
Maternal exposure to folic acid antagonists and placenta- mediated adverse pregnancy outcomes
}

\author{
Shi Wu Wen MB PhD, Jia Zhou MB MSc, Qiuying Yang MD PhD, William Fraser MD MSc, \\ Olufemi Olatunbosun MD, Mark Walker MSc MD
}

\section{ABSTRACT}

Background: In previous studies, maternal exposure to folic acid antagonists was associated with increased risks of neural tube defects, cardiovascular defects, oral clefts and urinary tract defects. The objective of the current study was to assess the possible effects of using folic acid antagonists in pregnancy on placenta-mediated adverse outcomes of pregnancy.

Methods: We used data from an administrative database to retrospectively compare the occurrence of placentamediated adverse pregnancy outcomes between pregnant women exposed to folic acid antagonists and women without exposure to these agents.

Results: We included in the analysis a total of 14982 women who had been exposed to folic acid antagonists and 59825 women who had not been exposed. Sulfamethoxazole-trimethoprim was the most frequently prescribed dihydrofolate reductase inhibitor (a total of 12546 exposures during the preconception period and all 3 trimesters), and phenobarbital was the most frequently prescribed among the other folic acid antagonists (a total of 1565 exposures). The risks of preeclampsia (adjusted odds ratio [OR] 1.52, 95\% confidence interval [CI] 1.39-1.66), severe preeclampsia (OR 1.77, $95 \% \mathrm{Cl}$ 1.38-2.28), placental abruption (OR 1.32, $95 \% \mathrm{Cl}$ 1.12-1.57), fetal growth restriction defined as less than the 10th percentile (OR 1.07, 95\% Cl 1.01-1.13), fetal growth restriction defined as less than the 3rd percentile (OR 1.22, $95 \% \mathrm{Cl} 1.11-1.34$ ) and fetal death (OR 1.35, 95\% Cl 1.07-1.70) were greater among mothers with exposure to folic acid antagonists. In general, the risks associated with exposure to other folic acid antagonists were higher than those associated with exposure to dihydrofolate reductase inhibitors. Supplementary analyses involving tight matching with propensity score, restriction of the analysis to women with exposure during the first and second trimesters and restriction of the analysis to specific categories of folic acid antagonists yielded similar results.

Interpretation: Maternal exposure to folic acid antagonists appears to increase the risk of placenta-mediated adverse outcomes of pregnancy.

Une version française de ce résumé est disponible à l'adresse www.cmaj.ca/cgi/content/full/179/12/1263/DC1

CMAJ 2008;179(12):1263-8

\section{Introduction}

Folic acid antagonists encompass a broad spectrum of drugs used for various clinical indications, including epilepsy, mood disorders and urinary tract infections. ${ }^{1}$ Folic acid antagonists can be divided into 2 loosely defined groups: the dihydrofolatereductase inhibitors, which block the conversion of folate to its more active metabolites, and other folic acid antagonists, a group consisting primarily of antiepileptic drugs (phenobarbital, phenytoin, primidone and carbamazepine) but also including Spasmophen (an antispasmodic drug that contains low doses of phenobarbital) and cholestyramine. ${ }^{1}$ According to the US Food and Drug Administration (FDA), many of the folic acid antagonists fall into that agency's pregnancy category $\mathrm{C}$ (drugs that should be given only if potential benefits outweigh potential risks to the fetus), pregnancy category $\mathrm{D}$ (drugs for which there is evidence of risks in pregnancy) or pregnancy category $\mathrm{X}$ (drugs for which there is evidence of clear risks in pregnancy). ${ }^{2}$

In a previous study, we found the following rates of folic acid antagonist use among women of reproductive age in any particular calendar year: $8.45 \%$ for dihydrofolate-reductase inhibitors and $1.14 \%$ for other folic acid antagonists. ${ }^{3}$ As such, a significant proportion of pregnancies probably involve exposure to folic acid antagonists, given that Pastuszak and associates ${ }^{4}$ reported that about half of all pregnancies in Canada and other industrialized countries were unplanned. Considering the potential of folic acid antagonists to deplete maternal folate and impair maternal folate metabolism, it is biologically plausible that maternal exposure to folic acid antagonists might cause adverse pregnancy outcomes, including adverse outcomes that have been hypothesized to share a common placenta-mediated pathway, such as preeclampsia, placental abruption, fetal growth restriction and fetal death. ${ }^{5-18}$ On the basis of these premises, we examined the effects of using folic acid antagonists in pregnancy on placenta-mediated adverse pregnancy outcomes.

From the OMNI (Obstetrics, Maternal, and Neonatal Investigation) Research Group (Wen, Zhou, Yang, Walker), Department of Obstetrics and Gynecology, and the Department of Epidemiology and Community Medicine (Wen, Walker), Faculty of Medicine, University of Ottawa, Ottawa, Ont.; the Clinical Epidemiology Program (Wen, Zhou, Yang, Walker), Ottawa Health Research Institute, Ottawa, Ont.; the School of Public Health (Zhou), Central South University, Changsha, Hunan, China; the Department of Obstetrics and Gynecology (Fraser), Faculty of Medicine, Université de Montréal, Montréal, Que.; and the Department of Obstetrics and Gynecology (Olatunbosun), Faculty of Medicine, University of Saskatchewan, Saskatoon, Sask. 


\section{Methods}

\section{Study design and data collection}

We conducted a retrospective population-based cohort study, using de-identified data from the linked maternal-infant database maintained for the Canadian province of Saskatchewan. We described details of the data set elsewhere. ${ }^{19}$ We identified all pregnant women with a singleton birth (both live births and stillbirths) in Saskatchewan from January 1, 1980, to December 31, 2000. Drug information was not available for the period from July 1, 1987, to December 31, 1988. We therefore excluded the births that occurred during this period or in the following year (i.e., until December 31, 1989). In addition, we excluded infants born to mothers with registered Indian status (about 18\% of infants), because drug information was not available for these mothers.

The "exposed" group consisted of mothers who had received prescriptions for folic acid antagonists during the 1-year period before delivery. We determined women's use of folic acid antagonists from information in the provincial outpatient prescription drug database, specifically the combination of gestational age, date of delivery and drug dispensing date. We included only folic acid antagonists that were dispensed in the 1-year period before delivery. For each woman who had been exposed to a folic acid antagonist in the 1-year period before delivery, we selected from the database 4 women who had not been exposed to these drugs, matched by infant's year of birth

Table 1: Baseline characteristics of women exposed and not exposed to folic acid antagonists during pregnancy in Saskatchewan between 1980 and 2000

\begin{tabular}{|c|c|c|}
\hline \multirow[b]{2}{*}{ Characteristic } & \multicolumn{2}{|c|}{ No. (\%) of participants* } \\
\hline & $\begin{array}{c}\text { Exposed } \\
n=14982\end{array}$ & $\begin{array}{c}\text { Not exposed } \\
n=59825\end{array}$ \\
\hline \multicolumn{3}{|l|}{ Maternal age, yr } \\
\hline$<20$ & $1637(10.9)$ & $4902 \quad(8.2)$ \\
\hline $20-29$ & $9820 \quad(65.5)$ & $39503(66.0)$ \\
\hline$\leq 30$ & 3525 (23.5) & $15420(25.8)$ \\
\hline Social assistance & $2083(13.9)$ & $5400 \quad(9.0)$ \\
\hline \multicolumn{3}{|c|}{ Type of hospital for birth } \\
\hline Provincial & 7428 (49.6) & $29702(49.6)$ \\
\hline Community & $4232(28.2)$ & $16879(28.2)$ \\
\hline Regional & 3204 (21.4) & 12789 (21.4) \\
\hline Unknown & 118 (0.8) & $455 \quad(0.8)$ \\
\hline \multicolumn{3}{|l|}{ Year of birth } \\
\hline 1980-1986 & $7810 \quad(52.1)$ & $33002(55.2)$ \\
\hline 1987-1991 & 1896 (12.7) & $7076(11.8)$ \\
\hline $1992-2001$ & $5276 \quad(35.2)$ & 19747 (33.0) \\
\hline Male & $7703 \quad(51.4)$ & 30628 (51.2) \\
\hline \multicolumn{3}{|l|}{ Parity } \\
\hline Primigravida & 6174 (41.2) & 22987 (38.4) \\
\hline Multipara & $8808 \quad(58.8)$ & 36838 (61.6) \\
\hline
\end{tabular}

*Unless otherwise indicated. (within 2 years), type of institution at birth (provincial, regional or community) and the first 3 digits of the mother's postal code. We obtained information on maternal demographic factors, including age, parity (number of live births) and provincial social assistance plan status, and neonatal characteristics, such as gestational age and birth weight, from provincial population registry and birth registration files.

\section{Outcomes}

We examined the following adverse outcomes of pregnancy: preeclampsia, severe preeclampsia, placental abruption, fetal growth restriction and fetal death. We used the International Classification of Diseases, 9th revision, to identify cases of preeclampsia (codes 642.4-642.7), severe preeclampsia (codes 642.5-642.7) and placental abruption (code 641.2). Although fetal growth restriction (also called "small-forgestational age") is usually defined as birth weight less than the 10th percentile, relative to the corresponding standard population stratum, ${ }^{20}$ we defined 2 levels of fetal growth restriction: birth weight less than the 3rd percentile and birth weight less than the 10th percentile, relative to the corresponding standard population stratum. Using 2 levels of fetal growth restriction allowed us to test the hypothesis that maternal exposure to folic acid antagonists might have a stronger effect on severe fetal growth restriction. We defined fetal death as intrauterine death occurring at gestational age greater than 20 weeks or weight greater than $500 \mathrm{~g}$.

\section{Statistical analysis}

We first compared the baseline characteristics of the women who had and had not been exposed to folic acid antagonists and described the distribution of the specific agents used. We then compared the rates of occurrence of adverse pregnancy outcomes between the 2 groups, for all folic acid antagonists and for the 2 subgroups of folic acid antagonists (dihydrofolatereductase inhibitors and other folic acid antagonists). We performed logistic regression analyses to examine the independent associations between exposure to folic acid antagonists and adverse pregnancy outcomes. We included the following independent variables in the regression models: use of folic acid antagonists, year of birth (1980-1986, 1987-1991 and 1992-2000, with 1992-2000 as the reference category), type of institution at birth (provincial, regional, community and unknown, with provincial as the reference category), maternal age $(<20,20-29$ and $\geq 30$ years, with 20-29 years as the reference category), parity (first v. second or higher order of birth, with second or higher order of birth as the reference category), social assistance (yes v. no, with no as the reference category) and infant's sex (male v. female, with male as reference category). We assessed the dose-response relation by classifying each woman's exposure into 1 of 3 levels, according to total quantity of folic acid antagonists dispensed $(<20,20-40$ or $>40$ tablets or capsules). We also performed supplementary analyses by tightly matching the data by propensity score (within 1 decimal point), by restricting the analysis to women who were exposed during the first and second trimesters only and by restricting the analysis to women who were exposed to 2 specific categories of folic acid antagonists (antibiotics for the 
treatment of urinary tract infection [sulfamethoxazole-trimethoprim] and antiepileptics [phenobarbital, phenytoin, carbamazepine, valproic acid, divalproex sodium]). Results are presented as odds ratios (OR) and $95 \%$ confidence intervals (CIs), where values greater than 1 signify a greater risk of the adverse outcome.

\section{Results}

We identified a total of 14982 women for whom folic acid antagonists were dispensed within the 1 -year period before delivery: 13396 who received dihydrofolate-reductase inhibitors and 1586 who received other folic acid antagonists. We also selected 59825 women for whom no folic acid antagonists were dispensed within the 1-year period before delivery; this number was 103 less than the planned ratio of $4: 1$ because we were unable to identify enough women who had not been exposed and who met the specified matching conditions.

The women who had been exposed to folic acid antagonists were younger, had lower parity and were more likely to be receiving social assistance (Table 1) than those who had not been exposed. Sulfamethoxazole-trimethoprim was the most frequently prescribed dihydrofolate-reductase inhibitor, and phenobarbital was the most frequently prescribed drug from among the other folic acid antagonists (Table 2). For most of the folic acid antagonists, prescriptions were more frequent during the preconception period and the first trimester than during the second and third trimesters, although there were some exceptions (e.g., triamterenehydrochlorothiazide and phenobarbital) (Table 2), especially in the earlier years of the study (see online Appendix 1, available at www.cmaj.ca/cgi/content/full/179/12/1263/DC2).

The risks of preeclampsia (especially severe preeclampsia), placental abruption, fetal growth restriction (especially severe fetal growth restriction) and fetal death were greater for mothers who had received prescriptions for folic acid antagonists during pregnancy (Figure 1). We observed a dose-response association for all of the outcomes examined (see online Appendix 2, available at www.cmaj.ca/cgi/content/full/179/12/1263/DC2).

Supplementary analyses examining the associations after tight matching by propensity score and after restricting the analysis to women with exposure during the first and second trimesters or to women who received specific categories of folic acid antagonists consistently showed greater risks of placenta-mediated adverse outcomes among the women who had been exposed to these drugs (see online Appendix 3, available at www.cmaj.ca/cgi/content/full/179/12/1263/DC2), although some fluctuations did occur for rare events.

\section{Interpretation}

In this population-based study, we found that women who were exposed to folic acid antagonists had greater risks of preeclampsia, placental abruption, fetal growth restriction and fetal death than women without such exposure. The risks associated with use of folic acid antagonists were higher for the severe forms of preeclampsia and fetal growth restriction, and we observed a dose-response association, related to the quantity of folic acid antagonists (as indicated by the number of tablets or capsules prescribed), for most of the outcomes. The results of various supplementary analyses, including tight matching by propensity score and restriction of the analyses to women with exposure during the first and second trimesters or to women with exposure to specific categories of folic acid antagonists, were consistent, demonstrating the robustness of the association. Our data also revealed the distribution of use of specific folic acid antagonists in routine practice, which represents useful feedback for clinicians.

\section{Strengths and limitations}

A major strength of our study is the testing of a novel hypothesis with a strong biologic rationale. Recent studies have sug- 
gested that folic acid supplementation in pregnancy may reduce the risk of placenta-mediated adverse outcomes such as preeclampsia. ${ }^{5-7}$ Ray and Laskin ${ }^{8}$ explored the potential mechanism of the relation between folic acid deficiency, hyperhomocysteine and the risk of placenta-mediated diseases, such as preeclampsia, placental abruption, fetal growth restriction and fetal death. The mechanism of placental abnormalities induced by folic acid deficiency and hyperhomocysteine is similar to the mechanism of folic acid deficiency and hyperhomocysteine in atherosclerosis and myocardial infarction in young women. ${ }^{9-18}$ Placental microvascular disease may arise from a maternal folate-homocysteine metabolic defect, a process that may start before pregnancy and continue throughout the entire pregnancy.

Our study was based on the entire population of Saskatchewan (where more than $90 \%$ of non-Aboriginal residents were eligible for provincial drug coverage); it therefore reflected routine practice in that province during the study period. This approach also reduced potential selection bias. The claims-processing systems in Saskatchewan had consistent data collection and coding procedures across different service sectors, along with checks for eligibility of benefits; ${ }^{21}$ these characteristics contributed to the quality of the database that we used. We used a recently developed Canadian standard to estimate fetal growth. ${ }^{20}$ The quality of the data for gestational age for recent years may have been better than that for previous years because of the increasing use of early ultrasound measurement to validate estimation by last normal menstrual period. ${ }^{22}$ Moreover, the developers of the new Canadian standard used a computer-assisted algorithm to correct gross errors in recorded gestational age..$^{20}$ As a result, we believe that our data on fetal growth restriction were robust.

The cohort study design allowed us to assess several outcomes simultaneously, which not only was a more comprehensive approach to our hypothesis but also helped us to assess the validity of the study findings. We used a combination of matching and multiple regression analyses to reduce potential confounding by maternal demographic and socioeconomic factors. The drug exposure information in our data did not depend on the woman's memory, which eliminated the possibility of recall bias. ${ }^{23} \mathrm{We}$ observed a consistent dose-response association for most of the outcomes we studied, which lends further support to a possible causative relation between exposure to folic acid antagonists and adverse pregnancy outcomes.

The most important limitation of studies of this nature is confounding by indication. For example, sulfamethoxazoletrimethoprim, the most frequently prescribed folic acid antagonist in this study, is usually prescribed to treat urinary tract infection. A recent systematic review and meta-analysis revealed that the risk of preeclampsia was greater among pregnant women with urinary tract infection (pooled OR 1.57,

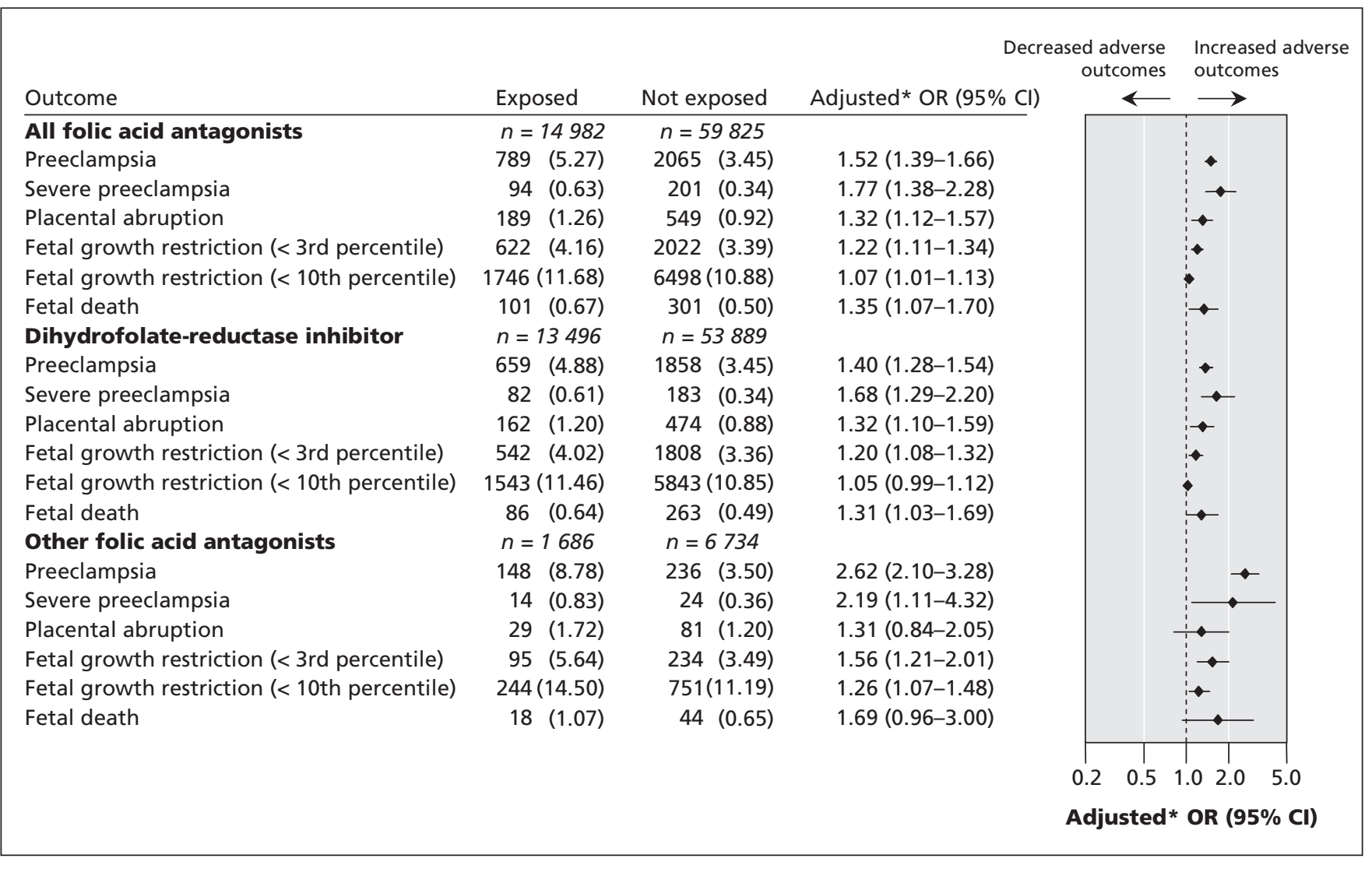

Figure 1: Comparison of adverse pregnancy outcomes between women exposed to folic-acid antagonists during pregnancy and women not exposed to these agents. *Adjusted for year of birth, type of institution at birth, maternal age, parity, social assistance and sex of the infant. 
95\% CI 1.45-1.70). ${ }^{24}$ As a result, it is difficult to determine whether the observed association between folic acid antagonists and adverse pregnancy outcome should be attributed to the prescribed drugs or to the underlying urinary tract infection. However, because a randomized controlled trial is neither ethical nor feasible in this situation, large cohort studies are the only way to investigate this type of research question. The folic acid antagonists that we studied are used for a wide range of clinical indications. The patient population is therefore heterogeneous, which would attenuate any specific relation between exposure and outcome. Moreover, results from a variety of sensitivity analyses yielded similar results, making it less likely that the observed associations were due to confounding by indication. Cohort studies comparing outcomes between women who took sulfamethoxazoletrimethoprim and women who took another antibiotic for urinary tract infection in pregnancy (e.g., amoxicillin) could help to reduce confounding by indication and generate more useful information for clinical practice.

For this study, we relied on an existing database. As a result, data for some important variables such as smoking, drinking and use of over-the-counter drugs (e.g., folic acid tablets) were not available, and we could not consider these factors in the data analysis. In our study, women who used folic acid antagonists had lower socio-economic status (as judged by the proportion who were receiving social assistance), which might lead to an increased risk of adverse pregnancy outcomes. ${ }^{25}$ Conversely, these women might have smoked more cigarettes during pregnancy, ${ }^{26}$ which might have decreased the risk of at least one of the outcomes we examined, preeclampsia. ${ }^{27}$ Thus, potential confounding between socio-economic status and cigarette smoking might have cancelled out the individual effects of these 2 factors, thereby limiting the effect of these factors on the observed association between folic acid antagonists and preeclampsia.

In addition, administrative data lack clinical details and are prone to a certain level of coding errors. ${ }^{28}$ Of particular concern is the potential for miscoding of preeclampsia and placental abruption in the databases. Furthermore, the Saskatchewan prescription drug file had no information about compliance. If the database included women who obtained folic acid antagonists from the pharmacy but did not actually ingest them, we would have misclassified them as having been exposed. We were unable to adjust for the potential problems caused by coding errors and noncompliance. However, because information in Saskatchewan's prescription drug file is obtained from pharmacists' dispensing records rather than physicians' prescription records, the effects of noncompliance may be limited. The only way to obtain data on actual drug consumption would be mothers' self-reporting. There is a trade-off between maternal self-reporting and the pharmacist's record. In a recent European study, mothers were able to report only $76 \%$ of the drug groups they consumed during pregnancy; when data on the exact name of the drug were sought, the figure dropped to $52 \% .{ }^{29}$ In the same study, of the drugs dispensed by the pharmacy, $6 \%$ were not used (i.e., noncompliance). In the study reported here, mis- classifications caused by either coding errors or noncompliance might have occurred randomly, which would tend to attenuate the observed effects. ${ }^{23}$

We identified the study cohort on the basis of both live births and stillbirths, which excluded, by definition, any pregnancies that were terminated because of fetal anomalies. Therefore, pregnancies that continued were those with a lower likelihood of harm based on these drug exposures. Another limitation of our study was the small proportion of cases for which there was a possibility that some of the drugs (e.g., triamterene-hydrochlorothiazide and phenytoin) were being used to treat pregnancy complications (e.g., preeclampsia), especially during the earlier years of the study.$^{30}$ Determining cause-effect relations would be difficult in these cases.

\section{Conclusion}

In this large population-based study, we found that maternal exposure to folic acid antagonists was associated with increased risks of several adverse pregnancy outcomes sharing a possible placenta-mediated cause. This finding, combined with previous findings on the association between exposure to folic acid antagonists during pregnancy and birth defects ${ }^{31,32}$ suggests the need to reclassify some of the folic acid antagonists in FDA pregnancy category $\mathrm{C}$ (e.g., sulfamethoxazoletrimethoprim) into FDA pregnancy category D. Physicians who are caring for women of reproductive age, particularly those already pregnant and those with medical conditions and a high risk of unintended pregnancy, should use caution in prescribing folic acid antagonists and should perhaps consider alternative agents as first-line therapy until further research confirms or refutes these findings. At a minimum, physicians should ensure that patients who need treatment with folic acid antagonists are fully informed about the potential risks associated with these drugs. Patients who do take these medications might benefit from increased supplemental folic acid (i.e., 5 mg by mouth once daily). ${ }^{31}$

This article has been peer reviewed.

Competing interests: None declared.

Contributors: Shi Wu Wen designed the study, obtained the funding, acquired the data and drafted the manuscript. Jia Zhou, Qiuying Yang, William Fraser, Olufemi Olatunbosun and Mark Walker made substantial contributions to the conception and design of the study. Mark Walker helped to acquire the data. Jia Zhou performed statistical analysis of the data and contributed to the interpretation of the results. Qiuying Yang and Mark Walker helped to analyze the data and interpret the results. William Fraser and Olufemi Olatunbosun helped to interpret the results. All of the authors revised the manuscript for important intellectual content and approved the version submitted for publication.

Acknowledgements: This study is based in part on nonidentifiable data provided by the Saskatchewan Department of Health. The interpretation and conclusions presented here do not necessarily represent those of the Government of Saskatchewan or the Saskatchewan Department of Health. Dr. Peter Garner (deceased) and Dr. Carl Nimrod (deceased) participated in the study design.

Shi Wu Wen is the recipient of an Ontario Women's Health Council-Canadian Institutes of Health Research (CIHR) Institute of Gender and Health Mid-Career Award and a research and development allowance from CIHR. Mark Walker is a CIHR New Investigator.

Funding: This study was funded by a grant from CIHR (MOP 67093). 


\section{REFERENCES}

1. Lambie DG, Johnson RH. Drugs and folate metablism. Drugs 1985;30:145-55.

2. US Food and Drug Administration, HHS. Requirements on content and format of labeling for human prescription drug and biological products. Fed Regist 2006;71:3921-97.

3. Wen SW, Walker M. Risk of fetal exposure to folic acid antagonists. J Obstet Gynaecol Can 2004;26:475-80.

4. Pastuszak A, Koren G, Milich V, et al. Prospective assessment of pregnancy outcome following first-trimester exposure to benzodiazepines. In: Koren G, editor. Maternal and fetal toxicology. New York (NY): Marcel Dekker; 1994. p. 77-88.

5. Bodnar LM, Tang G, Ness RB, et al. Periconceptional multivitamin use reduces the risk of preeclampsia. Am J Epidemiol 2006;164:470-7.

6. Hernandez-Diaz S, Werler MM, Louik C, et al. Risk of gestational hypertension in relation to folic acid supplementation during pregnancy. Am J Epidemiol 2002; 156:806-12.

7. Wen SW, Chen XK, Rodger M, et al. Folic acid supplementation in early second trimester and the risk of preeclampsia. Am J Obstet Gynecol 2008;198(1):45.e1-7.

8. Ray JG, Laskin C. Folic acid and homocycteine metabolic defects and the risk of placental abruption, pre-eclampsia and spontaneous pregnancy loss: a systematic review. Placenta 1999;20:519-29.

9. Graham IM, Daly LE, Refsum HM, et al. Plasma homocysteine as a risk factor for vascular disease. JAMA 1997;277:1775-81.

10. Rimm EB, Willett WC, Hu FB, et al. Folate and vitamin B6 from diet and supplements in relation to risk of coronary artery disease among women. JAMA 1998; 279:359-64.

11. Welch GN, Loscalzo J. Homocysteine and atherothrombosis. N Engl J Med 1998; 15:1042-50.

12. Schwartz SM, Siscovick DS, Malinow MR, et al. Myocardial infarction in young women in relation to plasma total homocysteine, folate, and a common variant in the methylenetetrahydrofolate reductase gene. Circulation 1997;96:412-7.

13. Chambers JC, McGregor A, Jean-Marie J, et al. Acute hyperhomocysteinaemia and endothelial dysfunction. Lancet 1998;351:36-7.

14. Ashworth JR, Warren AY, Johnson IR, et al. Plasma from pre-eclamptic women and functional change in myometrial resistance arteries. Br J Obstet Gynaecol 1998;105:459-61.

15. De Wolf F, Brosens I, Renaer M. Fetal growth retardation and the maternal arterial supply in the human placenta in the absence of sustained hypertension. Br J Obstet Gynaecol 1980;87:678-85.

16. Redman CWG. Current topic: pre-eclampsia and the placenta. Placenta 1991; 12:301-8.

17. Roberts JM, Taylor RM, Goldfein A. Clinical and biochemical evidence of endothelial cell dysfunction in the pregnancy syndrome preeclampsia. Am J Hypertens 1991;4:700-8.

18. Giugliani ERJ, Jorge SM, Concalves AL. Serum vitamin B12 levels in parturients, in the intervillous space of the placenta and in full-term newborns and their rela- tionships with folate levels. Am J Clin Nutr 1985;41:330-5.

19. Wen SW, Yang Q, Garner P, et al. Selective serotonin reuptake inhibitors and adverse pregnancy outcomes. Am J Obstet Gynecol 2006;194:961-6.

20. Kramer MS, Platt RW, Wen SW, et al.; Fetal/Infant Health Study Group of the Canadian Perinatal Surveillance System. A new and improved population-based Canadian reference for birth weight for gestational age. Pediatrics 2001;108:E35.

21. Downey W, Beck P, McNutt M et al. Health databases in Saskatchewan. In: Strom BL, editor. Pharmacoepidemiology. 3rd ed. Chichester (UK): Wiley; 2000. p. 325-45.

22. Kramer MS, McLean FH, Boyd ME, et al. The validity of gestational age estimation by menstrual dating in term, preterm, and postterm gestations. JAMA 1988; 260:3306-8.

23. Rothman KJ, Greenland S. Modern epidemiology. 2nd ed. Philadelphia (PA): Lippincott Raven Publishers; 1998.

24. Conde-Agudelo A, Villar J, Lindheimer M. Maternal infection and risk of preeclampsia: systematic review and metaanalysis. Am J Obstet Gynecol 2008; 198:7-22.

25. ACOG Committee on Practice Bulletins - Obstetrics. ACOG practice bulletion Diagnosis and management of preeclampsia and eclampsia. Number 33, January 2002. Obstet Gynecol 2002;99:159-67.

26. Krueger PM, Chang VW. Being poor and coping with stress: health behaviors and the risk of death. Am J Public Health 2008;98:889-96.

27. Conde-Agudelo A, Althabe F, Belizan JM, et al. Cigarette smoking during pregnancy and risk of preeclampsia: a systematic review. Am J Obstet Gynecol 1999; 181:1026-35.

28. Williams JI, Young W. A summary on the quality of health care administrative databases in Canada. In: Goel V, Williams JI, Anderson GM, et al., editors. Patterns of health care in Ontario. The ICES practice atlas. 2nd ed. Ottawa (ON): Canadian Medical Association; 1996.

29. De Jong van den Berg LT, Feenstra N, Sorensen HT, et al. Improvement of drug exposure data in a registration of congenital anomalies. Pilot-study: pharmacist and mother as sources for drug exposure data during pregnancy. European Medicine and Pregnancy Group. Teratology 1999;60:33-6.

30. Lucas MJ, Leveno KJ, Cunningham FG. A comparison of magnesium sulfate with phenytoin for the prevention of eclampsia. N Engl J Med 1995;333:201-5.

31. Hernandez-Diaz S, Werler MM, Walker AM, et al. Folic acid antagonists during pregnancy and the risk of birth defects. N Engl J Med 2000;343:1608-14.

32. Hernandez-Diaz S, Werler MM, Walker AM, et al. Neural tube defects in relation to use of folic acid antagonists during pregnancy. Am J Epidemiol 2001;153:961-8.

Correspondence to: Dr. Shi Wu Wen, OMNI Research Group,

Department of Obstetrics and Gynecology, Faculty of Medicine,

University of Ottawa, 501 Smyth Rd., Box 241, Ottawa ON K1H

8L6; fax 613 739-6266; swwen@ohri.ca

\begin{tabular}{|l|l|} 
Bulk reprints of $C M A J$ articles are available in minimum quantities of 50 \\
$\begin{array}{l}\text { For information or orders: } \\
\text { Reprint Coordinator } \\
\text { tel } 800 \text { 663-7336 } \mathrm{x} 2110, \text { fax } 613 \text { 565-7704 } \\
\text { janis.murrey @ cma.ca }\end{array}$
\end{tabular}

